\title{
Stability Behaviour of Monolayer Tetraether Lipids on the Amino-Silanised Silicon Wafer: Comparative Study between Langmuir-Blodgett Monolayers with Self-Assembled Monolayers
}

\author{
Sri Vidawati' ${ }^{1}$, Udo Bakowsky², Urich Rothe ${ }^{3}$ \\ ${ }^{1}$ Faculty of Post Graduate, National Institute of Science and Technology, Jakarta, Indonesia \\ ${ }^{2}$ Department of Pharmaceutics and Biopharmaceutics, Philipps University Marburg, Marburg, Germany \\ ${ }^{3}$ Institute of Physiological Chemistry, Faculty of Medicine, University Halle-Wittenberg, Hollystr, Halle/Saale, Germany \\ Email: sri.vidawati@istn.ac.id
}

How to cite this paper: Vidawati, S., Bakowsky, U. and Rothe, U. (2020) Stability Behaviour of Monolayer Tetraether Lipids on the Amino-Silanised Silicon Wafer: Comparative Study between Langmuir-Blodgett Monolayers with Self-Assembled Monolayers. Advances in Materials Physics and Chemistry, 10, 270-281.

https://doi.org/10.4236/ampc.2020.1011021

Received: September 11, 2020

Accepted: November 22, 2020

Published: November 25, 2020

Copyright $\odot 2020$ by author(s) and Scientific Research Publishing Inc. This work is licensed under the Creative Commons Attribution International License (CC BY 4.0).

http://creativecommons.org/licenses/by/4.0/ (c) (i) Open Access

\begin{abstract}
This study investigated the stability behaviour of molecular monolayer symmetric chemically modified tetraether lipids caldarchaeol- $\mathrm{PO}_{4}$ on the amino-silanised silicon wafer using Langmuir-Blodgett films, Self Assembling Monolayers (SAMs), ellipsometry, and atomic force microscopy (AFM). The monolayers of caldarchaeol- $\mathrm{PO}_{4}$ were stable on the solid surface amino-silanised silicon wafer. The organizations of molecular monolayers caldarchaeol- $\mathrm{PO}_{4}$ by Langmuir-Blodgett method and SAMs have been analyzed. The surface of pressure in Langmuir-Blodgett processing is carried out monolayers caldarchaeol- $\mathrm{PO}_{4}$ more flat island inhomogeneous. Another method of monolayers caldarchaeol- $\mathrm{PO}_{4}$ by SAMs is showed a large flat domain. Monolayers caldarchaeol- $\mathrm{PO}_{4}$ by Langmuir-Blodgett method seems to be stable and chemically resistant after washing with organic solvent and an additional treatment ultrasonification with various thickness lipids arround $2 \mathrm{~nm}$ to $6 \mathrm{~nm}$. Conversely, monolayer caldarchaeol- $\mathrm{PO}_{4}$ by SAMs appears fewer than monolayers caldarchaeol- $\mathrm{PO}_{4}$ by Langmuir-Blodgett method, the thickness of various from $1 \mathrm{~nm}$ to $3 \mathrm{~nm}$.
\end{abstract}

\section{Keywords}

Caldarchaeol- $\mathrm{PO}_{4}$, Langmuir-Blodgett Films, Self Assembling Monolayers (SAMs), Amino-Silanised Silicon Wafer

\section{Introduction}

The study of monolayers has quite implications in many fields of science and 
technology, such as in physics, material science, chemistry and biology. Molecular organization of organic monolayers on the solid surface can play an important role in interfacing bio-technological devices and many applications [1] [2] [3]. Monolayer bola amphiphiles have attracted the community for several years because these films provide a key model for understanding the molecular orientation and packing mode of bipolar molecules in the air-water interface. Self-assembled phospholipid membranes play an important role in biological processes. Mimicking these natural thin films, lipid-coated surfaces are of great importance to a number of biomedical applications, including intravenous drug delivery, biomaterials, and biosensors. Surface-supported lipid layers are also interesting as model surfaces in studies of protein adsorption or biophysical membrane studies.

A profound understanding of the behaviour of monolayer is crucial to exploiting the Langmuir-Blodgett monolayers. Preparation of arranged monolayers using Langmuir-Blodgett method is established on the organic molecules, such as lipids or phospholipids, to form a monolayer in the air-water interfaces. Monolayer stability and homogeneity are a prerequisite for the formation of organised lipid Langmuir-Blodgett films with excellent structural integrity. The organic monomolecular films have been shown to be useful for a variety of technological applications. The Langmuir-Blodgett film method has significant advantages in the study of the air-water and as air-solid interfacial behavior of thin solid films [4] [5] [6].

Self-assembly, in a general sense, may be defined as the spontaneous formation of the complex hierarchical structures of pre-designed building blocks, usually involving some energy scales and some degrees of freedom. The self-assembly is also a very general principle in nature, as seen in the formation of, for example, membranes of lipid molecules, or living cell as probably the most important paradigm. The self-assembled monolayers are ordered for the molecular assemblies that are formed spontaneously by the adsorption of a surfactant with the specific affinity of the headgroup to the substrate. Self assembly is a term of superamolecular chemistry [7] and is generally used to define the interaction of superamolecular among molecules in a solution or solid state to provide special arrangements or architectures. Self-assembled monolayers (SAMs) are highly ordered molecular assemblies formed spontaneously by chemisorptions and self organization of the long chain molecules on the surface of appropriate substrates. This is an interesting process for biological relevance as it provides a new approach to complex structures having nanoscale dimensions. Self-assembled monolayers (SAMs) comprise a fundamental of bottom-up nanotechnology widely used for applications such as interface control and solid surface functionalization.

The ability to mimic natural monolayers on lipid-coated surfaces is essential for a number of biomedical applications, such as intravenous drug delivery, as well as the production of biomaterials, and biosensors. Unfortunately, the extensive industrial application of the supported monomolecular lipid films has been 
prevented by the mechanical and chemical sensitivity of these thin layers. Studies of the organization of archaeal lipids in artificial membranes between two water environments indicate that in these systems lipids are generally organised as monolayers of fully expanded molecules, normal to the membrane fields [8]. Archaeal tetraether lipids can be of great value in bioelectronics, biomedicine and nanotechnology, because they can self-assemble into an ordered structure with tremendous stability against chemical degradation and microbes [9] [10] [11].

In this paper, we report the preparation of chemically modified symmetric tetraether lipids films caldarchaeol- $\mathrm{PO}_{4}$ in Figure 1. The film on the substrate prepared by Langmuir-Blodgett method and Self-assembled monolayers (SAMs) method. The amino-silanised substrate is prepared on the silicon wafer subtrates. Monolayer of caldarchaeol- $\mathrm{PO}_{4}$ on amino-silanised silicon wafer subtrates was subjected to rinsing with organic solvent and physical treatments (ultrasonification). We studied to analyze the behaviour comparisons between monolayers Langmuir Blodgett with SAMs of caldarchaeol- $\mathrm{PO}_{4}$ on the amino-silanised silicon wafers using AFM and ellipsometry.

\section{Material and Methods}

\subsection{Lipid and Other Materials}

The Lipids of the archae $S$. acidocaldarius is used for all of these experiments. Lipids is prepared from dried bacteria according to duPlesis (2008) [12]. Caldarchaeol is extracted and purified using the procedure Blöcher et al. (1984) [13]. A two-step chromatography method was developed, using DEAE-cellulose and silica columns, and the samples were eluted with chloroform and methanol. The head-groups (hydroxyl groups) of caldarchaeol are synthesized with $\mathrm{PO}_{4}$ according to the method of Bakowsky et al. (2006) [2]. The lipids are lyophilised at $10^{-2}$ Torr and stored obove phosphopentoxide. The monolayer experiments were carried out with the tetraether lipids from the highest purity available. All other chemicals obtained from Merck (Damstadt) or Sigma (Deisenhofen) at the highest purity.

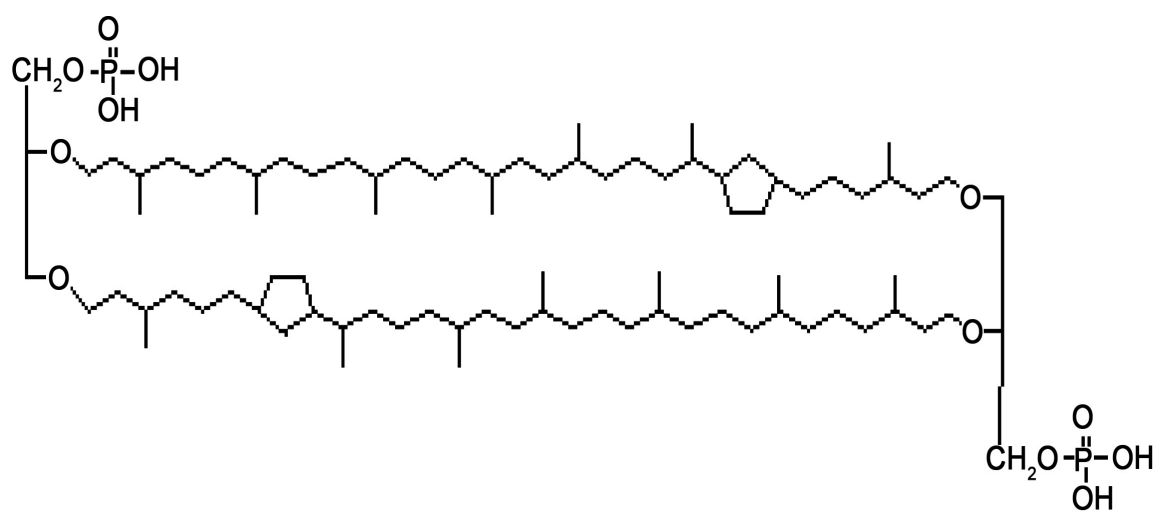

Figure 1. Caldarchaeol- $\mathrm{PO}_{4}$. 


\subsection{Silicon Wafers Subtrates}

Silicon wafers cut into dimensions $1.0 \mathrm{~cm} \times 1.0 \mathrm{~cm}$. We use the Piranha method to clean the silicon wafer substrates for all experiments. The Piranha solution, which is a mixture of concentrated sulfuric acid and hydrogen peroxide ( $7: 3$ concentrated $\mathrm{H}_{2} \mathrm{SO}_{4}: \mathrm{H}_{2} \mathrm{O}_{2}$ ), is a very common oxidizing agent used to clean silicon wafers substrate. The amino-silanised substrate is prepared on the silicon wafer subtrates. For activation, the wafer is placed under the UV-lamp (Xeradex, Lampenwerk Wipperfürth, Germany) for 5 minutes and then submersed in $1 \%$ aminopropyldimethylethoxysilane in chloroform at $70^{\circ} \mathrm{C}$ overnight. Amino-silanisation of the substrate produces a layer thickness of $1 \mathrm{~nm}$ and a water contact angle of $70^{\circ}$.

\subsection{Langmuir Blodgett Monolayer Experiments}

The investigations of the monolayer Langmuir is excreted by the commercial film balance ( $\mathrm{R} \& \mathrm{~K} \mathrm{GmbH}$, Mainz, Germany) by going a rectangular thermostatted Teflon trough (area $418 \mathrm{~cm}^{2}, 1 \mathrm{~cm}$ depth). The surface pressure is measured with a filter paper Wilhelmy balance. For the film balance experiments the tetraether lipids dissolved in chloroform/methanol 2:1 (v:v) consisting of a concentration $1.0 \mathrm{mmol} / \mathrm{l}$. Pure water of Milli Q quality (18.2 M, pH 5.6) was used as a subphase. All experiments were conducted at a temperature of $19^{\circ} \mathrm{C} \pm 1^{\circ} \mathrm{C}$. After spreading, the lipid films were equilibrated at zero surface pressure before starting the measurements. All films were compressed at a constant speed of $0.045 \mathrm{~cm}^{2} \cdot \mathrm{s}^{-1}\left(2.5 \times 10^{-2} \mathrm{~nm}^{2} \cdot \mathrm{s}^{-1}\right.$ per molecule). Y-type transfer (on the upward stroke) can be used on aminosilanized silcon wafers substrates.

\subsection{Self Assembly Monolayer Experiments}

The lipid monolayers are formed by self assembly. Material with an amino group on the surface silicon wafers treated with $\mathrm{PO}_{4}$ activated Caldarchaeol in acetone $\left(1 \mathrm{mg} / \mathrm{ml}\right.$ ) for 2 hours at $40^{\circ} \mathrm{C}$ in the presence of diisopropylethylamine.

\subsection{Ellipsometry Measurements}

The thicknesses of all monolayer films are characterized by ellipsometry. The ellipsometry measurement was performed by a mapping single wavelength of ellipsometer SE-400 (Sentech Instruments, Berlin, Germany). Such the light source, the $\mathrm{He} / \mathrm{Ne}$ laser with a wavelength of $1=623.8 \mathrm{~nm}$ was used. The incidence angle is $60^{\circ}$. For measurements, silicon wafers $(1 \mathrm{~cm} \times 1 \mathrm{~cm})$ with natural oxide layer (thickness $2.6 \mathrm{~nm}-2.9 \mathrm{~nm}$ ) and optical parameters of $\mathrm{ns}=3.828$ and $\mathrm{ks}=-\mathrm{i} 0.200$ are used. The refractive index of the tetraether lipids is established to be $\mathrm{nu}=1.58 \mathrm{As}$ a special scanning technique the line scan method is used for the presented measurements. Therefore, the substrates are covered only at the half of the total surface area by the lipid layer. The surface was scanned in a single line directing from the pure silicon oxide layer into the lipid layer. The distance between the measurement points was $200 \mathrm{~m}$ and the measured area of one 
detected point was $250 \mathrm{~m}^{2}$. For the correct determination of the mean film thickness an area of $1 \mathrm{~mm}^{2}$ in the middle of the lipid covered silicon wafer is scanned by mapping ellipsometry. The resolution was $250 \mathrm{~m}^{2}$ per scanned point and the presented values are calculated according to the statistical standard deviation method using all points in the observed area.

\subsection{Atomic Force Microscopy Measurements}

The morphology of the monolayer film surface is characterized using an Atomic force microscopy. The Atomic force microscopy is performed using NanoWizard apparatus (JPK instruments, Berlin, Germany) under atmospheric conditions. The commercial pyramidal $\mathrm{Si}_{3} \mathrm{~N}_{4}$ tips (NSC16AlBS, Micromasch, Estonia) are mounted on cantilever with a length of $230 \mu \mathrm{m}$, a resonance frequency of approximately $170 \mathrm{kHz}$ and a nominal force constant of approximately $40 \mathrm{~N} / \mathrm{m}$ used. Scan the size and scan frequency using from 0.5 to $1.5 \mathrm{~Hz}$. AFM image characterization is accomplished by indicating the signal height in the retrace direction $(512 \times 512$ pixels $)$.

\section{Result}

\subsection{Tetraether Lipid Caldarchaeol- $\mathrm{PO}_{4}$ Monolayers on the Amino-Silanised Silicon Wafer}

According Vidawati et al. [14] [15] [16] the Langmuir film monolayer of caldarchaeol- $\mathrm{PO}_{4}$ is performed thermodynamic stable. The isothermal surface is generally used to characterize the strength and stability of LB-films. On the surface of pressure/area $(\pi / \mathrm{A})$ isotherm caldarchaeol- $\mathrm{PO}_{4} \mathrm{LB}$-films are most stable at a surface pressure of about $18 \mathrm{mN} / \mathrm{m}$, the molecular area between $1.0 \mathrm{~nm}^{2}$ and $1.5 \mathrm{~nm}^{2}$ in concentration caldarchaeol- $\mathrm{PO}_{4}$ of $30 \mathrm{~L} 1 \mathrm{mMol}$ at the air-water interface. This parameter has been used in the experiment of LB-monolayer of caldarchaeol-PO ${ }_{4}$. LB-monolayer was deposited using Y-type film transfers for amino-silanised silicon wafer.

The other lipid monolayers are formed by self assembly. Material with amino-silanised silicon wafer was treated with $\mathrm{PO}_{4}$ activated Caldarchaeol in acetone $(1 \mathrm{mg} / \mathrm{ml})$ for 2 hours at $40^{\circ} \mathrm{C}$ in the presence of diisopropylethylamine. The silicon wafer is than washed with chloroform and methanol.

\subsection{Ellipsometry}

The thickness of all monolayer of caldarchaeol- $\mathrm{PO}_{4}$ is characterized by ellipsometry. Ellipsometric data provides the mean film thickness over a large spot area of $250 \mathrm{~m}^{2}$, and therefore cannot distinguish between films with different morphological structures. The lipids are observed after transfer onto the amino-silanised substrates and the thickness values are recorded. The thickness of $0.8 \mathrm{~nm}$ for the amino-silanised surface.

The monolayer of caldarchaeol- $\mathrm{PO}_{4}$ on the amino-silanised silicon wafer is then washed with organic solvent and the thickness of each substrate is again 
measured. Finally, the monolayers of caldarchaeol- $\mathrm{PO}_{4}$ on the amino-silanised silicon wafer are subjected to additional ultrasonification treatment, to analyze the resistance and stability of the films on the amino-silanised surface.

\subsection{Atomic Force Microscopy}

The morphology of the surface and thickness of monolayer caldarchaeol- $\mathrm{PO}_{4}$ studied on a molecular scale using AFM. The Typical topographical images of the AFM of the monolayer caldarchaeol- $\mathrm{PO}_{4}$ on the amino-silanised surfaces are rinsed with organic solvents and is subjected them to ultrasonification treatment displayed in Figure 2. To further analyse the behavior of monolayers caldarchaeol- $\mathrm{PO}_{4}$ comparison between by Langmuir Blodgett method and SAMs, the area in the middle of AFM image has been scratched with the AFM-tip, All images displayed in Figure 3.

\section{Discussion}

Lipid monolayers were formed by self-assembly and Langmuir-Blodgett methods. In the first step, an inert silicone surface should be activated. A very popular substrate for silane-based SAMs and Langmuir-Blodgett monolayer is silicon with its native oxide layer. In this case, the comparison of the structure and morphology is less a problem, since the native oxide layer is non-crystalline but usually very smooth. However, a specific cleaning procedure should be applied. For the removal of possible organic contaminants from the substrate as well as the glassware used for solution deposition, "piranha solution" is often used. The amino-silanised substrate was prepared on $\mathrm{SiO}_{2}$ wafer subtrates. For activation, the wafer was placed under the UV-lamp
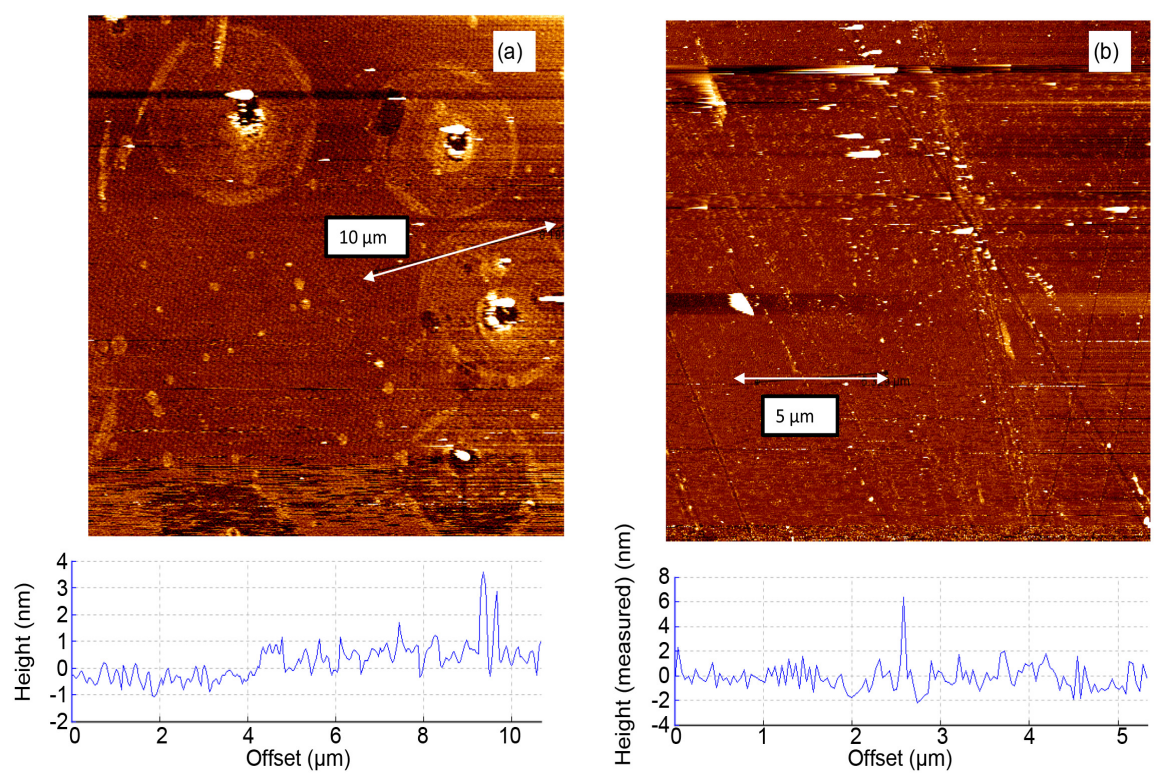

Figure 2. Representative AFM images of monolayer caldarchaeol- $\mathrm{PO}_{4}$ after washing with organic solvent and an additional treatment ultrasonification (a) Langmuir-Blodgett method, (b) Self-Assembled Monolayers (SAMs) method. 

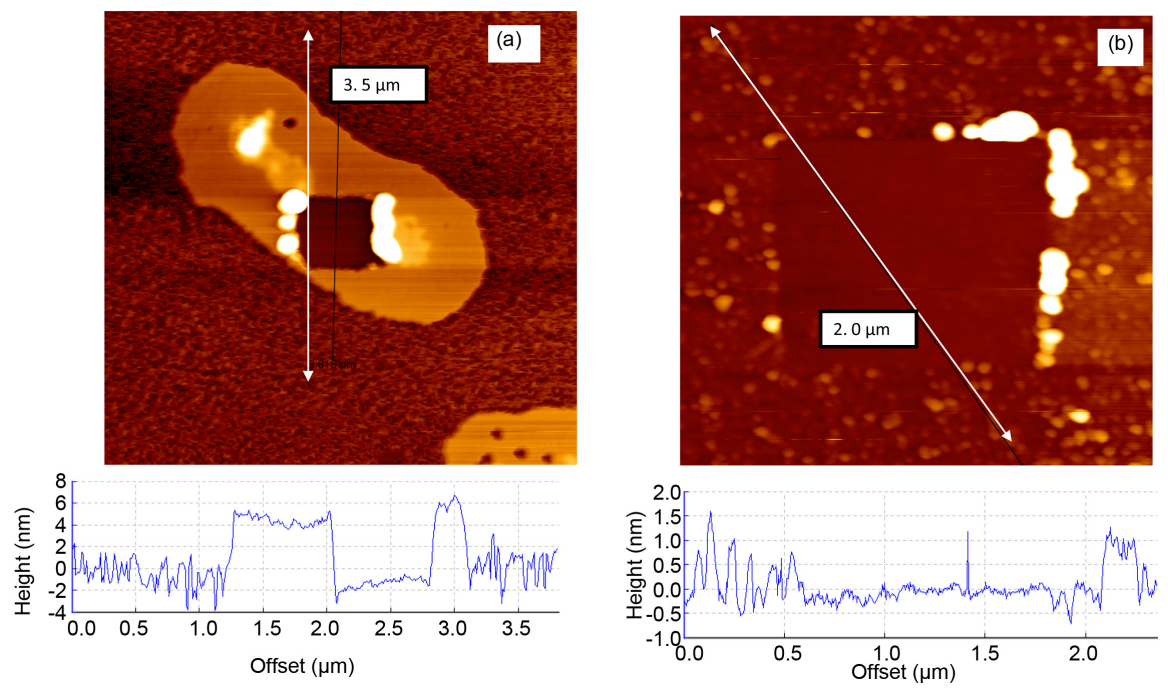

Figure 3. Representative AFM images with scratched in the middle area with AFM-tip of monolayer caldarchaeol- $\mathrm{PO}_{4}$ after washing with organic solvent and an additional treatment ultrasonification (a) Langmuir-Blodgett method, (b) Self-Assembled Monolayers (SAMs) method.

(Xeradex, Lampenwerk Wipperfürth, Germany) for 5 minutes and then submersed in $1 \%$ aminopropyldimethyle-thoxysilane in chloroform at $70^{\circ} \mathrm{C}$ overnight. Amino-silanised of the substrate produced a layer thickness of $1 \mathrm{~nm}$ and a water contact angle of $70^{\circ}$. Silanization was confirmed by FTIR measurements. Thus it was possible to attach a dense monomolecular lipid layer on the silicone surface and to provide a sufficient amount of chemical binding sites for further modification strategies. The molecules introduced in this step were chemically coupled into the second phosphate group on the caldarchaeol, and these molecules preferentially had to contain amino groups.

The self-assembly, in a general sense, can be defined as the spontaneous formation of the complex hierarchical structures of the previously designed building blocks, usually involving some energy scales and some degrees of freedom. Self-assembly is also a very common principle in nature, as seen in the formation of, for example, membranes from lipid molecules, or the living cell is probably the most important paradigm. The self-assembled monolayers are ordered molecular assemblies that are formed spontaneously by the adsorption of a surfactant with the specific affinity of the headgroup to the substrate [17].

On the other hand, based on the experimental experience with a simple SAM system, the concept of SAMs is ready to be utilized for the applications of monolayers with pre-designed properties. These range from the use of SAMs as building blocks for heterostructures to wetting control and for specific adsorption of biomolecules. With a variety of fundamental problems and, at the same time, very diverse applications, the importance of SAMs is derived less than the usefulness of a particular system, but rather from the general concept SAMs. We believe that this concept will prove important and beneficial to many technical applications as well as in the view of its role as a low-dimensional molecular 
model of the system.

The Langmuir films consist of an amphiphilic molecule that spreads on liquid surface such as water [18]. The hydrophilic headgroup has an affinity for the water while the end group is a hydrophobic stick out on the other side. The Langmuir-Blodgett (LB) films were prepared by transferring the Langmuir films to a solid substrate [19] [20]. Multilayers are prepared by repeated immersion (periodic) on the substrate in the proper solutions.

In the Langmuir-Blodgett method, caldarchaeol- $\mathrm{PO}_{4}$ has a collapse pressure of $18 \mathrm{mN} / \mathrm{m}$ and a molecular area between $1.0 \mathrm{~nm}^{2}$ and $1.5 \mathrm{~nm}^{2}$ [15]. Ellipsometric measurements after a transfer to an amino-silanised surface by Langmuir-Blodgett method is a small domain with a thickness between $2.0 \mathrm{~nm}$ and $4.5 \mathrm{~nm}$ has been formed. We conclude that the caldarchaeol- $\mathrm{PO}_{4}$ can be transferred to the amino-silanised, the film adopts both upright standing and horseshoe configurations, which can be attributed to the charge interactions of the ammino-modified surface and the lipid headgroups. Another method by SAMs, monolayers of caldarchaeol- $\mathrm{PO}_{4}$ has been formed thickness arround $5.0 \mathrm{~nm}$ and 6.0 $\mathrm{nm}$, the films adopted an upright standing configuration.

AFM was also employed to observe surface morphology. Monolayer films on the surface of amino-silanised by Langmuir-Blodgett method and SAMs were analyzed. The stability of archaebacterial membranes has been attributed with their composition and structure on an interface, where monolayers are the only stable structure; and the archael membranes can be prepared by Langmuir-Blodgett method [14] [21] [22]. SAMs have been successfully formed from a modified tetraether lipids (caldarchaeol- $\mathrm{CyCl}$ ) on a silicon surface, and a new process for coating silicon surfaces with caldarchaeol monolayers has been developed, with special emphasis on coating applications for catheters and other medical devices [23] [24]. Structural surface analysis and interfacial features are crucial to comparing and understanding the properties of these nano systems. The adsorption and stability of the monolayers used for solid surfaces modification are important to prepare and control the properties of the tetraether membrane lipid films for various applications.

Most of our AFM results for the monolayers of the caldarchaeol- $\mathrm{PO}_{4}$ on an amino-silanised surface indicate that most of the lipids are removed after washing with organic solvent and an additional treatment ultrasonification. For the monolayers of caldarchaeol- $\mathrm{PO}_{4}$ by Langmuir-Blodgett method on an amino-silanised surface, AFM image analysis is revealed, along with many small circular domains, than appeared to be inhomogeneous flat islands with a thickness of arround $2.0 \mathrm{~nm}$ to $6.0 \mathrm{~nm}$, see in Figure 3(a). After transfer to the amino-silanised monolayers of caldarchaeol- $\mathrm{PO}_{4}$ are subjected to rinsing with organic solvent (chloroform) and physical treatment of ultrasonification. For monolayer caldarchaeol- $\mathrm{PO}_{4}$ by SAMs on an amino-silanised surface, AFM image analysis reveals a large flat domain, between $2.0 \mathrm{~nm}$ to $4.0 \mathrm{~nm}$, see in Figure 3(b). Monolayers of caldarchaeol- $\mathrm{PO}_{4}$ by Langmuir-Blodgett method more 
thickness and stabilize than the monolayers of caldarchaeol- $\mathrm{PO}_{4}$ by SAMs after washing with organic solvent and an additional treatment ultrasonification.

To further analyze the behavior of monolayers caldarchaeol- $\mathrm{PO}_{4}$ comparison with Langmuir Blodgett method and SAMs, the area in the middle of the AFM image has scratched with AFM-tip. Monolayers caldarchaeol- $\mathrm{PO}_{4}$ by Langmuir-Blodgett method seems to be stable and chemically resistant with various thickness lipids arround $2 \mathrm{~nm}$ to $6 \mathrm{~nm}$ see in Figure 3(a). Conversely monolayers caldarchaeol- $\mathrm{PO}_{4}$ by SAMs appear fewer then monolayers caldarchaeol- $\mathrm{PO}_{4}$ by Langmuir-Blodgett method, the thickness of various from $1 \mathrm{~nm}$ to $3 \mathrm{~nm}$ see in Figure 3(b).

We can see the difference between the Langmuir Blodgett monolayer and SAMs is not completely significant. The Langmuir layers are on liquid surfaces and are usually also weakly bound to the substrate. SAMs as we define them here are on solid substrates and chemisorbed, which is strongly bounded. An interesting intermediate case would be a molecule on the liquid substrate with a strong interaction. The organizations of molecular monolayers Tetraether lipids by Langmuir-Blodgett method and SAMs have been analyzed [15] [21] [23]

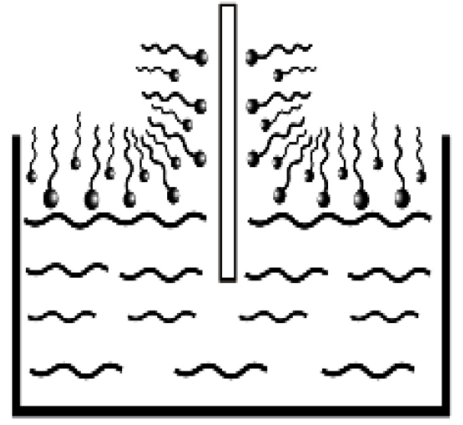

(a)

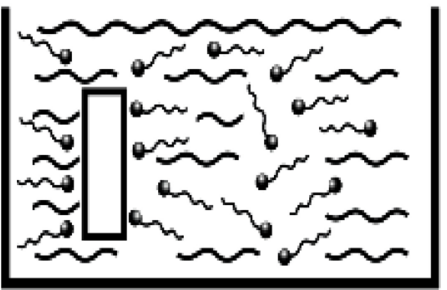

(b)

Figure 4. Organization molecule of monolayers Tetraether lipids by (a) Langmuir-Blodgett method and (b) Self-Assembled Monolayers (SAMs) method [15] [21] [23].

Thickness of monolayer caldarchaeol $\mathrm{PO}_{4}(\mathrm{~nm})$

by Ellipsometry and AFM

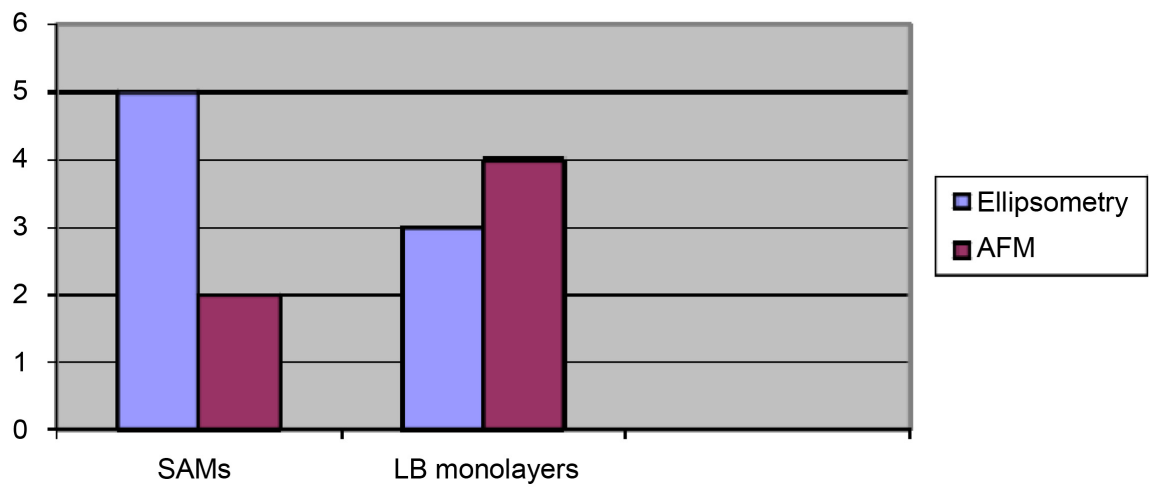

Figure 5. Comparison thickness of monolayers caldarchaeol- $\mathrm{PO}_{4}$ between Langmuir-Blodgett method and Self-Assembled Monolayers (SAMs) method. 
in Figure 4. The comparison thickness of monolayer caldarchaeol PO4 by ellipsometry and AFM measurement can see in Figure 5. The surface of pressure in Langmuir-Blodgett proceesing is carried out monolayers caldarchaeol- $\mathrm{PO}_{4}$ more flat island inhomogeneous on the amino-silanised silicon wafer. Another method of monolayers caldarchaeol- $\mathrm{PO}_{4}$ by SAMs is showed a large flat domain on the amino-silanised silicon wafer.

\section{Acknowledgements}

This study was supported by the Deutscher Akademischer Austauschdienst (DAAD, SV).

\section{Conflicts of Interest}

The authors declare no conflicts of interest regarding the publication of this paper.

\section{References}

[1] Weissbuch, I., Baxter, P.N.W., Cohen, S., Cohen, H., Kjaer, K., Howes, P.B., Als-Nielsen, J., Hanan, G.S., Schubert, U.S., Lehn, J.-M., Leiserowitz, L. and Lahav, M.J. (1998) Self-Assembly at the Air-Water Interface. In-Situ Preparation of Thin Films of Metal Ion Grid Architectures. Journal of the American Chemistry Society, 120, 4850-4860. https://doi.org/10.1021/ja980205w

[2] Bakowsky, U., Kneuer, C., Rothe, U., Liefeith, K., Frant, M., Dölling, K., Schmid, R., Johnsen, H. and Stenstad, P. (2006) Patent PCT/DE2005/001162.

[3] Swalen, J.D., Allara, D.L., Andrade, J.D., Chandross, E.A., Garoff, S., Israelachvili, J., McCarthy, T.J., Murray, R., Pease, R.F., Rabolt, J.F., Wynne, K.J. and Yu, H. (1987) Molecular Monolayers and Films. A Panel Report for the Materials Sciences Division of the Department of Energy. Langmuir, 3, 932-950.

https://doi.org/10.1021/la00078a011

[4] Love, J.C., Estroff, L.A., Kriebel, J.K., Nuzzo, R.G. and Whitesides, G.M. (2005) Self-Assembled Monolayers of Thiolates on Metals as a Form of Nanotechnology. Chemical Reviews, 105, 1103-1170. https://doi.org/10.1021/cr0300789

[5] Shirahata, N., Hozumi, A. and Yonezawa, T. (2005) Monolayer-Derivative Functionalization of Non-Oxidized Silicon Surfaces. The Chemical Record, 5, 145. https://doi.org/10.1002/tcr.20041

[6] Fendler, J.H. (2001) Chemical Self-Assembly for Electronic Applications. Chemstry of Materials, 13, 3196-3210. https://doi.org/10.1021/cm010165m

[7] Lehn, J.-M. (1995) Supramolecular Chemistry. VCH, Weinheim. https://doi.org/10.1002/3527607439

[8] Gambacorta, A., Gliozzi, A. and De Rosa, M. (1995) Archaeal Lipids and Their Biotechnological Applications. World Journal of Microbiology and Biotechnology, 11, 115-131. https://doi.org/10.1007/BF00339140

[9] Hanford, M.J. and Peeples, T.L. (2002) Archaeal Tetraether Lipids Unique Structures and Applications. Applied Biochemistry and Biotechnology, 97, 45-62. https://doi.org/10.1385/ABAB:97:1:45

[10] De Rosa, M., Morana, A., Riccio, A., Gambacorta, A., Trincone, A. and Incani, O. 
(1994) Lipids of the Archaea: A New Tool for Bioelectronics. Biosensors and Bioelectronics, 9, 669-675. https://doi.org/10.1016/0956-5663(94)80064-2

[11] Freisleben, H.-J. (2019) The Main (Glyco) Phospholipid (MPL) of Thermoplasma acidophilum. International Journal of Molecular Sciences, 20, 5217.

https://doi.org/10.3390/ijms20205217

[12] Bode, M.L., Buddoo, S.R., Minnaar, S.H. and Du Plessis, C.A. (2008) Extraction, Isolation and NMR Data of the Tetraether Lipid Calditoglycerocaldarchaeol (GDNT) from Sulfolobus metallicus Harvested from a Bioleaching Reactor. Chemistry and Physics of Lipids, 154, 94-104.

https://doi.org/10.1016/j.chemphyslip.2008.02.005

[13] Blöcher, D., Gutermann, R., Henkel, B. and Ring, K. (1984) Physicochemical Characterization of Tetraether Lipids from Thermoplasma acidophilum Differential Scanning Calorimetry Studies on Glycolipids and Glycophospholipids. Biochimica et Biophysica Acta (BBA)-Biomembranes, 778, 74-80. https://doi.org/10.1016/0005-2736(84)90449-8

[14] Vidawati, S., Sitterberg, J., Bakowsky, U. and Rothe, U. (2010) AFM and Ellipsometric Studies on LB Films of Natural Asymmetric and Symmetric Bolaamphiphilic Archaebacterial Tetraether Lipids on Silicon Wafers. Colloids and Surfaces B: Biointerfaces, 78, 303-309. https://doi.org/10.1016/j.colsurfb.2010.03.015

[15] Vidawati, S., Sitterberg, J., Rothe, U. and Bakowsky, U. (2011) Stability of Monomolecular Films of Archaebacterial Tetraether Lipids on Silicon Wafers: A Comparison of Physisorbed and Chemisorbed Monolayers. Colloids and Surfaces B: Biointerfaces, 87, 209-216. https://doi.org/10.1016/j.colsurfb.2011.05.005

[16] Vidawati, S., Rothe, U. and Bakowsky, U. (2012) Langmuir Films Stability Phenomenon of Glycerol Dialkyl Nonitol Tetraether at the Air-Water Interface for Variations Spreading Time. Advances in Biological Chemistry, 2, 233-237. https://doi.org/10.4236/abc.2012.23028

[17] Menger, F.M., Richardson, S.D. and Bromley, G.R. (1989) Ion Conductance along Lipid Monolayers. Journal of the American Chemical Society, 11Ž, 68936894.

[18] Kaganer, V.M., Möhwald, H. and Dutta, P. (1999) Structure and Phase Transitions in Langmuir Monolayers. Reviews of Modern Physics, 71, 779. https://doi.org/10.1103/RevModPhys.71.779

[19] Ulman, A. (1991) An Introduction to Ultrathin Organic Films: From Langmuir-Blodgett to Self-Assembly. Academic Press, Boston. https://doi.org/10.1016/B978-0-08-092631-5.50009-9

[20] Vidawati, S. and Rothe, U. (2015) Monolayers Langmuir-Blodgett Films of Synthetic Artificial Mimic Molecules That Resemble the Following Tetraether Lipids on Silicon Wafers. Advances in Biological Chemistry, 5, 189-196. https://doi.org/10.4236/abc.2015.54015

[21] Bakowsky, U., Rothe, U., Antonopoulos, E., Martini, T., Henkel, B. and Freisleben, H.J. (2000) Monomolecular Organization of the Main Tetraether Lipid from Thermoplasma acidophilum at the Water-Air Interface. Chemistry and Physics of Lipids, 105, 31-42. https://doi.org/10.1016/S0009-3084(99)00131-0

[22] Kim, J.M. and Thompson, D.H. (1992) Tetraether Bolaform Amphiphiles as Models of Archaebacterial Membrane Lipids: Synthesis, Differential Scanning Calorimetry, and Monolayer Studies. Langmuir, 8, 637-644.

https://doi.org/10.1021/la00038a056

[23] Frant, M., Stenstad, P., Johnsen, H., Dölling, K., Rothe, U., Schmid, R. and Liefeith, K. (2006) Anti-Infective Surfaces Based on Tetraether Lipids for Peritoneal Dialysis 
Catheter Systems. Matwiss. u. Werkstofftech, 37, 6.

[24] Frant, M., Dayyoub, E., Bakowsky, U. and Liefeith, K. (2018) Evaluation of a Ureteral Catheter Coating by Means of a BioEncrustation in Vitro Model. International Journal of Pharmaceutics, 546, 86-96. https://doi.org/10.1016/j.ijpharm.2018.04.023 